\title{
A NEW INTERPRETATION OF THE LOW RADIAL VELOCITY DETERMINED FROM THE HEII EMISSION LINE IN THE GALACTIC SUPERSOFT X-RAY SOURCE RX J0019.8+2156
}

\author{
Izumi Hachisu $^{1}$ and Mariko Kat $)^{2}$
}

We propose a new interpretation for the low radial velocity of $\mathrm{He}$ II emission line in the Galactic supersoft X-ray source RX J0019.8 +2156 based on a $2-D$ numerical simulation of dynamical mass transfer in binary systems. A more massive main-sequence (MS) companion of $\sim 2.0 M_{\odot}$ is derived for a $\sim 0.6 M_{\odot}$ white dwarf (WD) primary.

The Galactic supersoft X-ray source RX J0019.8+2156 (hereafter, RX J0019) with a $12.2 \mathrm{mag}$ optical counterpart was discovered by ROSAT. The source is a binary with the period of 0.66 days and its X-ray luminosity is fairly constant, at least, for $7 \mathrm{yrs}$. Therefore RX J0019 seems to be a typical luminous supersoft X-ray source (SSS) with steady hydrogen shell-burning on a mass-accreting white dwarf (WD). In such SSSs, the companion transfers mass to the WD primary at a rate of $\gtrsim 1 \times 10^{-7} M_{\odot} \mathrm{yr}^{-1}$ or more. Such a high rate is realized when the companion is more massive than the WD. However, recent observations indicate low radial velocities of He II emission lines (from the WD or disk ?), suggesting a less massive companion.

We propose a new interpretation for the low radial velocity of He II emission line in RX J0019. A more massive main-sequence (MS) companion of $\sim 2.0 M_{\odot}$ is derived for a $\sim 0.6 M_{\odot}$ white dwarf (WD) primary, contrary to the recent estimate of the very low mass companion of $0.3-0.5 M_{\odot}$, which is derived from the low radial velocity semiamplitude of $K \sim 50-70 \mathrm{~km} \mathrm{~s}^{-1}$ for the He II $\lambda 4686$ emission line. We have simulated dynamical flows in mass-transferring binary systems and found that the emission line comes from the heated surface of the companion near the $L_{1}$ point and does not follow the motion of the primary as shown in. Figure 1. We may conclude that the companion is as massive as $q=M_{\mathrm{MS}} / M_{\mathrm{WD}} \sim 3-4$ for $M_{\mathrm{WD}} \sim 0.6 M_{\omega}$. Our irradiated $\mathrm{L}_{1}$ point model also provides natural explanations for various observational features of emission lines.

\footnotetext{
${ }^{1}$ Department of Earth Science and Astronomy, University of Tokyo, Japan 153-8902 (hachisu@chianti.c.u-tokyo.ac.jp).

${ }^{2}$ Department of Astronomy, Keio University, Japan 2238521 (mariko@educ.cc.keio.ac.jp).
}

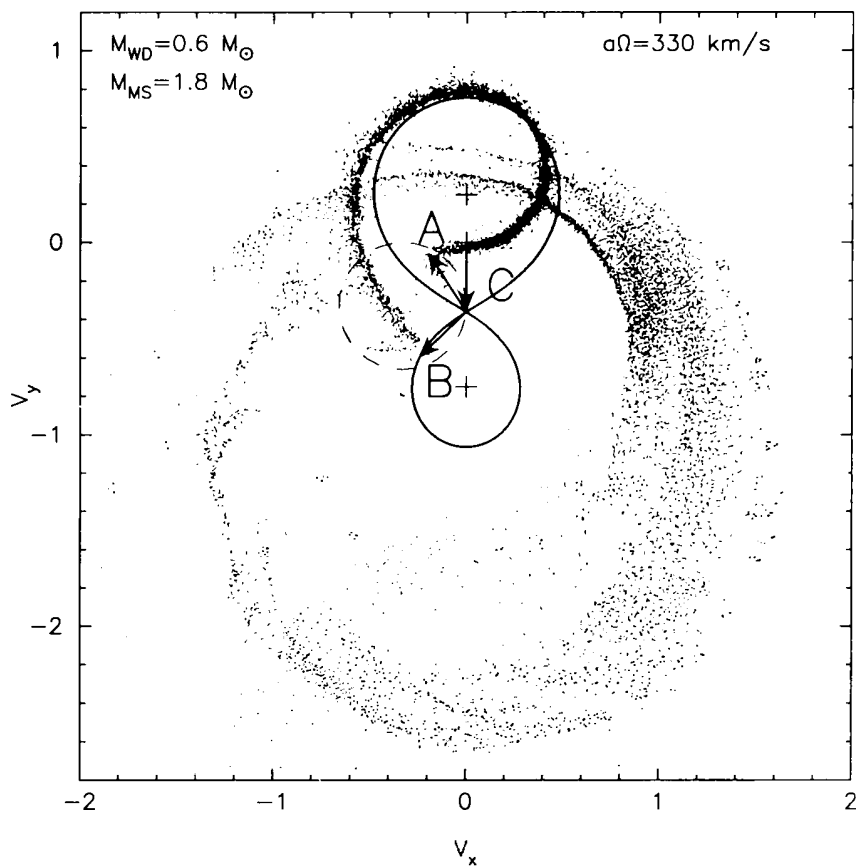

Fig. 1. Doppler tomogram for the case of $M_{W 1}=0.6 M$ and $M_{\mathrm{MS}}=1.8 M_{\odot}$ in units of orbital velocity, i.e.. $a \Omega_{\mathrm{orb}}=330 \mathrm{~km} \mathrm{~s}^{-1}$ : the velocity of the Roche lobes (thick solid curves), the center of mass for the mainsequence companion (top cross), the center of mass for the binary system (middle cross), and the position of the white dwarf (bottom cross). Thick concentration of mass particles around the upper Roche lobe corresponds to the surface of the MS component whereas broad circular disk-like thin concentration of particles centered at the lower Roche lobe represents the flow of the accretion disk. Arrow A represents the flow velocity on the right side of the $\mathrm{L}_{1}$ point while arrow $\mathrm{B}$ does the flow velocity on the left side of the $L_{1}$ point. Arrow $C$ is the rotational velocity of the $\mathrm{L}_{1}$ point itself. The observed intensity of the He II $\lambda 4686$ emission has a peak around $V_{X} \sim-100 \mathrm{~km} \mathrm{~s}^{-1}$ and $V_{Y} \sim-100 \mathrm{~km} \mathrm{~s}^{-1}$. which is denoted by a dashed circle 